\title{
Atorvastatin alleviates renal ischemia-reperfusion injury in rats by promoting M1-M2 transition
}

\author{
QI WANG, YAN-YAN SU, YAN-QUN LI, YUN-FANG ZHANG, SHEN YANG, \\ JIE-LIN WANG and HONG-YAN LI
}

\begin{abstract}
Department of Nephrology, Huadu District People's Hospital, Southern Medical University, Guangzhou, Guangdong 510800, P.R. China
\end{abstract}

Received September 7, 2015; Accepted September 19, 2016

DOI: $10.3892 / \mathrm{mmr} .2016 .6074$

\begin{abstract}
Acute kidney injury (AKI) often occurs as a result of ischemia-reperfusion (IR). Previous studies have demonstrated that inflammation is an important contributor to AKI. Atorvastatin (ATO) possesses anti-inflammatory properties and has been demonstrated to exert protective effects against renal IR injury (IRI). However, the underlying mechanism requires further study. In the present study, a rat model of renal IRI was successfully established. Consistent with the results of a previous study, ATO significantly attenuated IRI, which was supported by a decrease in serum creatinine and an increase in creatinine clearance rate, as well as alleviated pathological alterations in renal tubular cells. There are two types of activated macrophages: Proinflammatory M1 and anti-inflammatory M2 macrophages, which have been demonstrated to exert contributory and protective effects on IRI, respectively. The present study demonstrated that treatment with ATO significantly decreased M1 macrophage density and increased M2 macrophage density, as compared with the IR group. In addition, it is well known that M1 macrophages can be induced by $\mathrm{T}$ helper 1 cytokines, including tumor necrosis factor (TNF)- $\alpha$ and interferon (IFN)- $\gamma$, whereas M2 macrophages can be induced by peroxisome proliferator-activated receptor (PPAR) $-\gamma$. The present study indicated that ATO treatment significantly decreased the expression levels of TNF- $\alpha$ and IFN- $\gamma$, and increased PPAR- $\gamma$ expression. In conclusion, ATO may ameliorate renal IRI by promoting M1-M2 transition. Furthermore, ATO-mediated macrophage polarization in rats with renal IRI may be associated with the downregulation of TNF- $\alpha$ and IFN- $\gamma$, and the upregulation of PPAR- $\gamma$.
\end{abstract}

Correspondence to: Dr Hong-Yan Li, Department of Nephrology, Huadu District People's Hospital, Southern Medical University, 48 Xinhua Road, Guangzhou, Guangdong 510800, P.R. China E-mail: lihy0726@126.com

Key words: atorvastain, ischemia-reperfusion injury, tumor necrosis factor- $\alpha$, interferon- $\gamma$, peroxisome proliferator-activated receptor- $\gamma$, macrophage activation

\section{Introduction}

Acute kidney injury (AKI) is a common disease, associated with a high morbidity and mortality; the reported incidence of AKI varies from 5\% of all hospitalized patients to $30-50 \%$ of patients in intensive care units (1). At present, no therapeutic agents have been proven to prevent AKI (2). Kidney ischemia-reperfusion (IR) is known to be a major cause of AKI. Ischemic injury is present in $\sim 50 \%$ of patients with AKI (3) and is induced by several factors, including hypotension, hypoperfusion, hypoxia, oxidative stress and renal vasoconstriction (4). Methods to ameliorate IR injury (IRI) may provide an effective therapeutic strategy for the treatment or prevention of ischemic AKI.

It is well known that inflammation serves an important role in AKI. IR has previously been shown to initiate pathogenesis in vascular endothelial cells and tubular epithelial cells, and may result in a loss of immune system homoeostasis in the kidney (2). Macrophages belong to the family of mononuclear phagocytes and perform various critical roles in homeostasis, surveillance, immune response, and tissue injury and repair $(5,6)$. Macrophages have been reported to importantly contribute to ischemic AKI. In addition, macrophages have been identified as important regulators of wound healing from primarily initiating an inflammatory response, to later facilitating tissue repair and downregulating inflammation (7). The diverse functions of macrophages are attributed to the plasticity of the macrophage phenotype, which includes classically activated macrophages (M1) and alternatively activated macrophages (M2) (8). M1 macrophages secrete various proinflammatory mediators and have been reported to serve critical roles in some inflammatory diseases, including inflammatory bowel disease, atherosclerosis and insulin resistance in obesity (9-11). Conversely, M2 macrophages counterbalance M1-induced inflammation and promote tissue repair $(12,13)$.

Atorvastatin (ATO) is one of the most effective pharmaceutical agents for the treatment of cardiovascular disease. In addition to its lipid lowering properties via the inhibition of 3-hydroxy-3-methylglutaryl coenzyme A reductase, ATO also possesses several pleiotropic effects, including anti-inflammatory and antioxidant properties, thus modulating effects on endothelial function and vascular wall structure (14). It has 
previously been reported that treatment with ATO protects the kidney from IRI in rats, and promotes human monocyte differentiation toward M2 macrophages $(15,16)$.

The present study aimed to investigate whether ATO has the capacity to facilitate rat monocyte differentiation toward M2 macrophages in a rat model of renal IRI. In addition, the underlying mechanisms were investigated.

\section{Materials and methods}

Animals. Male Sprague-Dawley rats (weight, 200-250 g; age, 7 weeks), were purchased from Guangzhou University of Chinese Medicine Laboratory Animal Center (Guangzhou, China). All animal procedures conducted in the present study were performed strictly in accordance with the recommendations of the National Institutes of Health Guide for the Care and Use of Laboratory Animals (1996) and the present study was approved by Huadu District People's Hospital (Guangzhou, China). The rats had ad libitum access to food and water, were housed under controlled conditions of $23 \pm 3^{\circ} \mathrm{C}$ room temperature and $55 \pm 15 \%$ relative humidity, and were maintained under a $12 \mathrm{~h}$ light-dark cycle.

The 40 rats were randomly assigned to the following four groups $(n=10)$ : IR + ATO group, rats with IRI were administered a single intravenous dose of ATO $(10 \mathrm{mg} / \mathrm{kg}) 30 \mathrm{~min}$ prior to reperfusion; IR group, rats with IRI were administered intravenous saline; sham group, sham-operated rats were administered intravenous saline; and control group, rats were administered intravenous saline. Saline was administered at the same volume as the ATO.

To generate the IRI model, surgery was conducted as previously described (17). Briefly, following administration of general anesthesia (3\% napental at $30 \mathrm{mg} / \mathrm{kg}$ ), the rats underwent bilateral incisions and the right kidney was removed. The left renal artery was exposed and was then occluded for $60 \mathrm{~min}$. Subsequently, the incisions were closed to allow the rats to recover and the organ was allowed to reperfuse for $24 \mathrm{~h}$. The sham group underwent the same operation; however, the left renal artery was not clamped.

Serum preparation and tissue collection. All rats were sacrificed $\sim 24 \mathrm{~h}$ after surgery by injection by $3 \%$ sodium pentobarbital $(30 \mathrm{mg} / \mathrm{kg})$. Blood samples were collected via cardiac puncture and were left to clot at room temperature. Following centrifugation at $3,000 \times \mathrm{g}$ for $5 \mathrm{~min}$ at $4^{\circ} \mathrm{C}$, the extracted serum samples were pipetted into a clean tube and were stored at $-80^{\circ} \mathrm{C}$ until further use. After collecting blood samples from the heart, the kidneys were immediately dissected and were preserved in $10 \%$ neutral buffered formalin for further immunohistological examinations. During the experiment, urine samples were collected using metabolic cages.

Measurement of serum creatinine (Scr) and creatinine clearance rate $(\mathrm{Ccr})$. The levels of Scr and urinary creatinine were determined using an automatic biochemistry analyzer (ADVIA 1800; Siemens AG, Munich, Germany). Based on the levels of urinary creatinine, Scr, urine volume and body weight, $\mathrm{Ccr}$ was calculated according to the following formula (18): $\mathrm{Ccr}=$ urinary creatinine $(\mu \mathrm{mol} / \mathrm{l}) \times$ urinary volume $(\mathrm{ml} / \mathrm{kg} / \mathrm{min}) / \mathrm{Scr}(\mu \mathrm{mol} / \mathrm{l})$.
Histological examination. The fixed kidneys were cut into transverse sections and embedded in paraffin. Sections $(\sim 4 \mu \mathrm{m})$ were prepared and processed for staining with hematoxylin and eosin $(0.1 \%$ hematoxylin for $6 \mathrm{~min}$ at room temperature; $0.5 \%$ eosin for $2 \mathrm{~min}$ at room temperature), periodic acid-Schiff (oxidized in $0.5 \%$ periodic acid solution for $5 \mathrm{~min}$ at room temperature; Schiff reagent for $15 \mathrm{~min}$ at room temperature) and periodic acid-methenamine silver (oxidised in $0.5 \%$ periodic acid solution for $15 \mathrm{~min}$ at room temperature; methenamine silver working solution for $1 \mathrm{~h}$ at $60^{\circ} \mathrm{C}$ ). Renal tubular injuries, including renal necrosis, brush border detachment, tubular cast formation and tubular dilation, were analyzed at 20 randomly selected high power fields under light microscopy (magnification, 400x) in the renal outer medulla. According to the ratio of tubular injury, the degrees of tubular injury were scaled from $0-5$, as follows: 0 , not present; $1, \leq 10 \%$; $2,11-25 \% ; 3,26-50 \% ; 4,51-75 \% ; 5, \geq 76 \%$.

Terminal deoxynucleotidyl transferase dUTP nick end labeling (TUNEL) assay. The 4- $\mu \mathrm{m}$ sections underwent TUNEL staining using the In Situ Apoptosis Detection kit (Roche Diagnostics, Indianapolis, IN, USA) according to the manufacturer's protocol, to determine the rate of apoptosis in the kidney. Briefly, after dewaxing and dehydrating, the sections were incubated with protease for $30 \mathrm{~min}$ at $37^{\circ} \mathrm{C}$ and then with TUNEL reaction mixture for $\sim 20 \mathrm{~min}$ at $37^{\circ} \mathrm{C}$ in the dark. Following treatment with converter-peroxidase solution at $37^{\circ} \mathrm{C}$ for $10 \mathrm{~min}$ in a moisture chamber, the sections were incubated with 3,3'-diaminobenzidine solution for 5-6 min at room temperature. Apoptotic cells were characterized by dark brown staining in the nuclei. Each section was examined at six randomized high power fields under a fluorescence microscopy (magnification, 400x).

ELISA. TNF- $\alpha$ and IFN- $\gamma$ concentrations were determined in the collected serum samples using Rat TNF- $\alpha$ Quantikine ELISA kit and Rat IFN- $\gamma$ Quantikine ELISA kit (R\&D Systems, Inc., Minneapolis, MN, USA) according to the manufacturer's protocols. Three independent tests were conducted.

Immunofluorescence staining. Frozen sections were prepared and immunohistofluoresence analysis was conducted using specific primary antibodies against rat cluster of differentiation (CD)68 (1:100; Santa Cruz Biotechnology, Inc., Dallas, TX, USA; cat. no. sc-70760), CD206 (1:100; Santa Cruz Biotechnology, Inc.; cat. no. sc-34577), inducible nitric oxide synthase (iNOS; 1:100; eBioscience, Inc., San Diego, CA, USA; cat. no. 17-5920-80) and PPAR- $\gamma$ (1:400; Santa Cruz Biotechnology, Inc.; cat. no. sc-6284) overnight at $4^{\circ} \mathrm{C}$. In addition, CD68/iNOS, CD68/CD206 and CD68/PPAR- $\gamma$ double staining was conducted by incubating sections at 1:100 dilutions with the antibodies overnight at $4^{\circ} \mathrm{C}$. 4',6-Diamidino-2-phenylindole (DAPI) was used to detect nucleate cells under a fluorescence microscope.

Statistical analysis. Results are presented as the mean \pm standard deviation. Data from the different groups were compared using one-way analysis of variance followed by Tukey's post-hoc analysis with SPSS 22.0 (IBM SPSS, Armonk, NY, 
Table I. Extent of kidney injury in control, sham, IR and $\mathrm{IR}+$ ATO rats.

\begin{tabular}{lcccccc}
\hline & \multicolumn{5}{c}{ Number of rats with tubular injury } \\
\cline { 2 - 7 } Group & 0 & 1 & 2 & 3 & 4 & 5 \\
\hline Control & 5 & 5 & 0 & 0 & 0 & 0 \\
Sham & 3 & 7 & 0 & 0 & 0 & 0 \\
IR & 0 & 2 & 4 & 1 & 3 & 0 \\
IR + ATO & 1 & 3 & 3 & 3 & 0 & 0
\end{tabular}

IR, ischemia-reperfusion; ATO, atorvastatin. Histological evaluation of kidney injury was conducted $24 \mathrm{~h}$ after renal IR. The severity of injury was graded as follows: 0 , not present; $1, \leq 10 \% ; 2,11-25 \% ; 3$, $26-50 \% ; 4,51-75 \% ; 5, \geq 76 \%$.

A

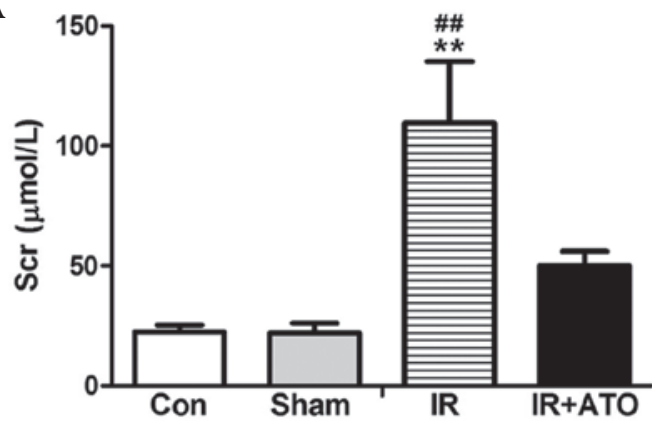

B

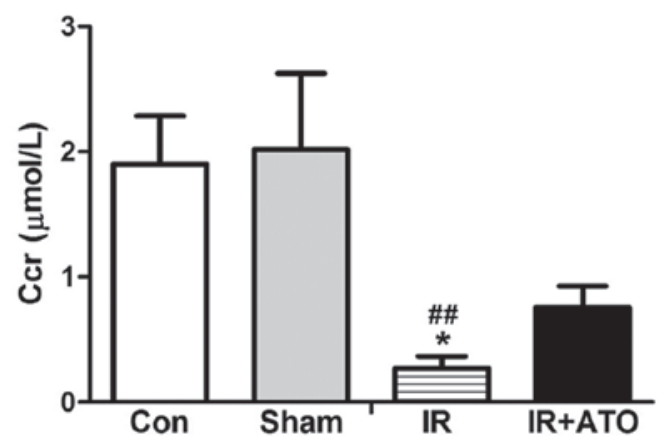

Figure 1. Effects of ATO on Scr levels and Ccr in IR rats. Rats in the $\mathrm{IR}+\mathrm{ATO}$ group were treated with a single intravenous dose of $10 \mathrm{mg} / \mathrm{kg}$ ATO followed by IR surgery. Blood samples were collected to obtain serum for the measurement of (A) Scr and (B) Ccr. Data are presented as the mean \pm standard deviation, $n=6$. ${ }^{*} \mathrm{P}<0.05$ vs. IR + ATO group; ${ }^{* *} \mathrm{P}<0.01$ vs. IR + ATO group; ${ }^{\# \#} \mathrm{P}<0.01$ vs. Con group, as determined by analysis of variance. IR, ischemia-reperfusion; ATO, atorvastatin; Con, control; Scr, serum creatinine; Ccr, creatinine clearance rate.

USA). $\mathrm{P}<0.05$ was considered to indicate a statistically significant difference.

\section{Results}

ATO treatment significantly alleviates renal IRI. To evaluate the effects of ATO on IRI, Scr levels were determined in the rats $24 \mathrm{~h}$ after IR. As shown in Fig. 1, Scr levels were significantly increased and the Ccr was significantly decreased in the IR group, compared with in the control and sham-operated groups. The Scr levels and the Ccr were comparable between the sham and control groups. These results indicate that the rat model of renal IRI was successfully established. In addition, for the IR + ATO group, the ATO treatment relatively alleviated the Scr increase and the Ccr decrease compared with the IR group. Renal pathological alterations were evaluated in the rats $24 \mathrm{~h}$ after IR. Histologically, $24 \mathrm{~h}$ after IR, the control rats developed granular and vacuolar degeneration in renal tubular epithelial cells, tubular casts, broader brush detachment and tubular dilation (Fig. 2). Following ATO treatment, the aforementioned pathological alterations were markedly alleviated. In addition, no lesions were observed in kidneys of sham-operated rats. Kidney injury severity was graded among the groups (Table I).

Further analysis by TUNEL assay revealed that IRI was associated with the increased apoptosis of tubular epithelium cells (Fig. 3). However, following treatment with ATO, IR-induced apoptosis was markedly suppressed. These results indicate ATO treatment may significantly attenuate renal IRI.

ATO treatment decreases serum TNF- $\alpha$ and IFN- $\gamma$ levels. To determine the effects of ATO on proinflammatory cytokine levels, the serum levels of TNF- $\alpha$ and IFN- $\gamma$ were detected $24 \mathrm{~h}$ after IR. As shown in Fig. 4, the serum levels of TNF- $\alpha$ and IFN- $\gamma$ in the sham group were comparable to the control group. However, the levels were significantly increased in the IR group compared with the sham group. Following treatment with ATO, the levels were significantly decreased compared with the IR group. These results suggest that ATO treatment may significantly inhibit IRI-induced inflammation.

ATO treatment accelerates M2 macrophage polarization. To evaluate the effects of ATO on macrophage activation, M1 and M2 macrophages were detected by double immunofluorescence staining. Macrophages were detected by red fluorescence staining with an anti-CD68 antibody (macrophage marker); M1 macrophages were detected by green fluorescence staining with an anti-iNOS antibody; and M2 macrophages were detected by green fluorescence staining with an anti-CD206 antibody. Compared with the sham group, the number of M1 macrophages was markedly increased, whereas the number of M2 macrophages was markedly decreased in IR group. Conversely, M1 and M2 macrophages in the IR + ATO group were markedly decreased and increased, respectively, compared with the IR group (Fig. 5). These results indicate that ATO may promote the M1 to M2 transition in rats with IRI.

ATO treatment increases PPAR- $\gamma$ expression in renal macrophages. The effects of ATO on PPAR- $\gamma$ expression in macrophages were investigated. Using double immunofluorescence staining, renal tissues were analyzed for PPAR- $\gamma$ (green) and CD68 (red). As shown in Fig. 6, nucleate cells in the renal tissues were labeled with DAPI. Compared with the sham group, PPAR- $\gamma$ expression was markedly decreased in the IR group. Conversely, PPAR $-\gamma$ expression in macrophages was increased in the IR + ATO group compared with the control group, and was markedly increased compared with the IR group. These results suggest that ATO may induce PPAR- $\gamma$ expression in macrophages. 
A
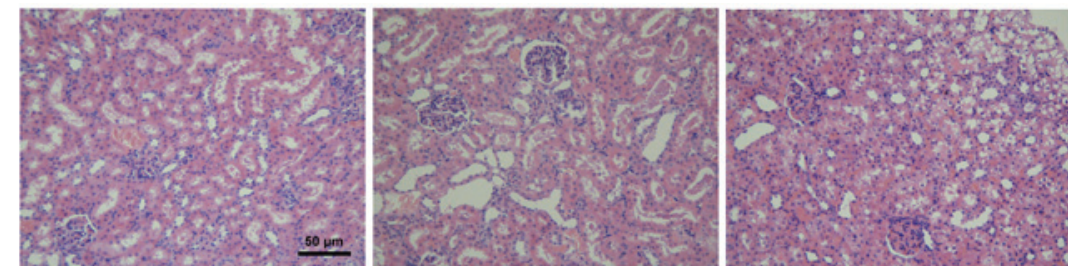

B
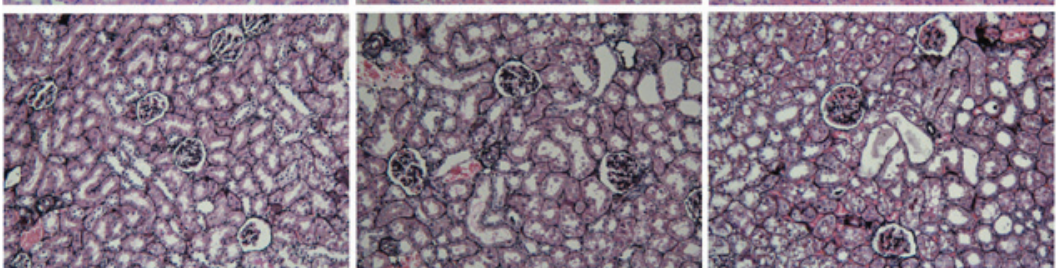

C
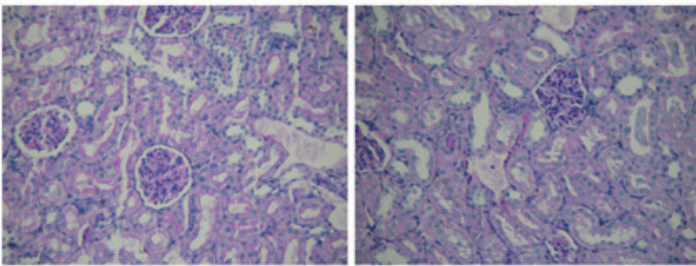

IR

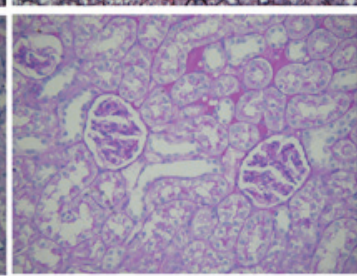

Sham

IR+ATO

Figure 2. Effects of ATO on kidney histological features of rats. Rats were sacrificed $24 \mathrm{~h}$ after ATO administration. Kidney samples from the sham group, IR group and IR + ATO group were collected to perform (A) hematoxylin and eosin, (B) periodic acid-Schiff and (C) periodic acid-methenamine silver staining. Scale bar, $50 \mu \mathrm{m}$. IR, ischemia-reperfusion; ATO, atorvastatin.

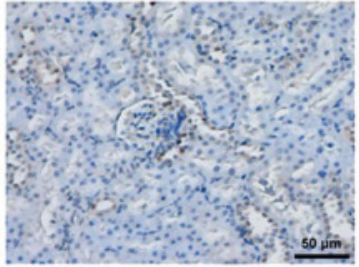

Sham

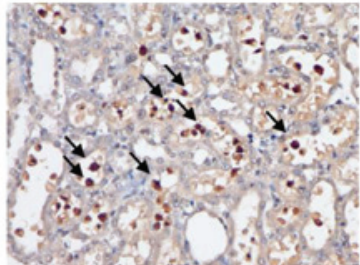

IR

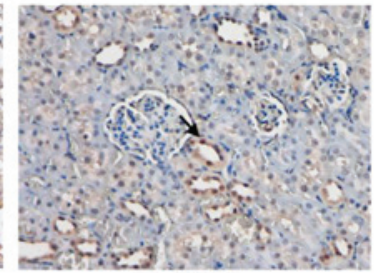

IR+ATO

Figure 3. In situ termimal deoxynucleotidyl transferase dUTP nick end labeling staining of rat kidney samples. Dark brown staining (arrows) indicates positive staining of apoptotic cells. Scale bar, $50 \mu \mathrm{m}$. IR, ischemia-reperfusion; ATO, atorvastatin.
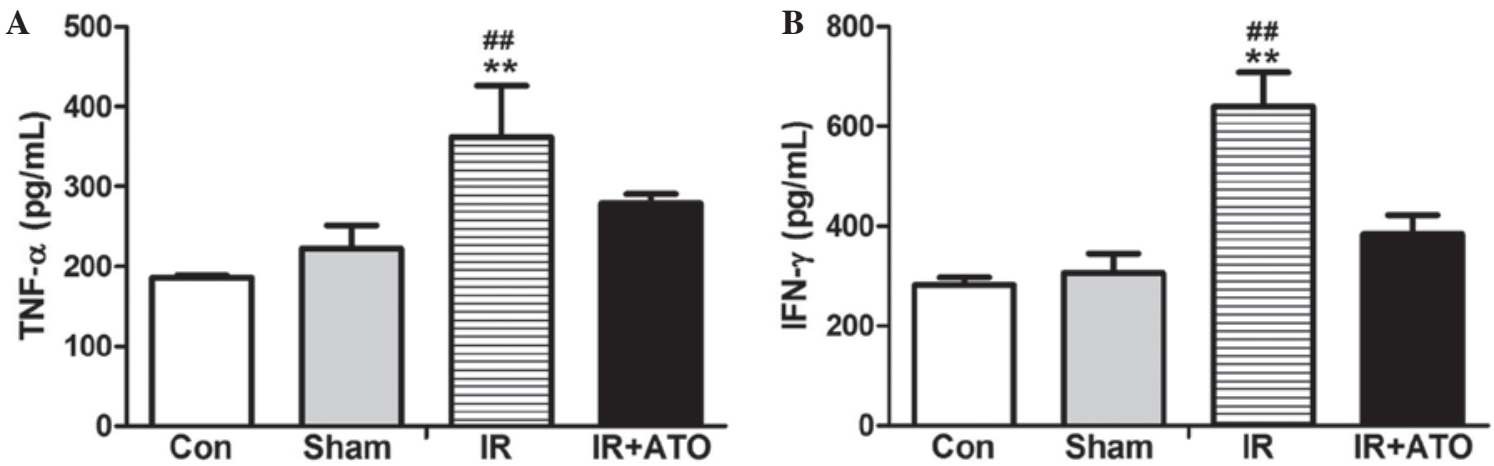

Figure 4. Effects of ATO on TNF- $\alpha$ and INF- $\gamma$ levels in IR rats. Rats in the IR + ATO group were treated with a single intravenous dose of $10 \mathrm{mg} / \mathrm{kg}$ ATO 30 min followed by IR surgery. Blood samples were collected to obtain serum for measurement of (A) TNF- $\alpha$ and (B) INF- $\gamma$. Data are presented as the mean \pm standard deviation, $\mathrm{n}=6 .{ }^{* * *} \mathrm{P}<0.01$ vs. IR $+\mathrm{ATO}$ group; ${ }^{\# *} \mathrm{P}<0.01$ vs. Con group, as determined by analysis of variance. IR, ischemia-reperfusion; ATO, atorvastatin; Con, control; TNF- $\alpha$, tumor necrosis factor- $\alpha$; IFN- $\gamma$, interferon- $\gamma$.

\section{Discussion}

There is an urgent requirement for the identification of therapeutic strategies that protect the kidney from IRI during surgery or transplantation. The present study confirmed that treatment with ATO could attenuate renal IRI. Furthermore, the results of the present study demonstrated that ATO treatment induced PPAR- $\gamma$ expression and activation in rat kidney samples, leading 


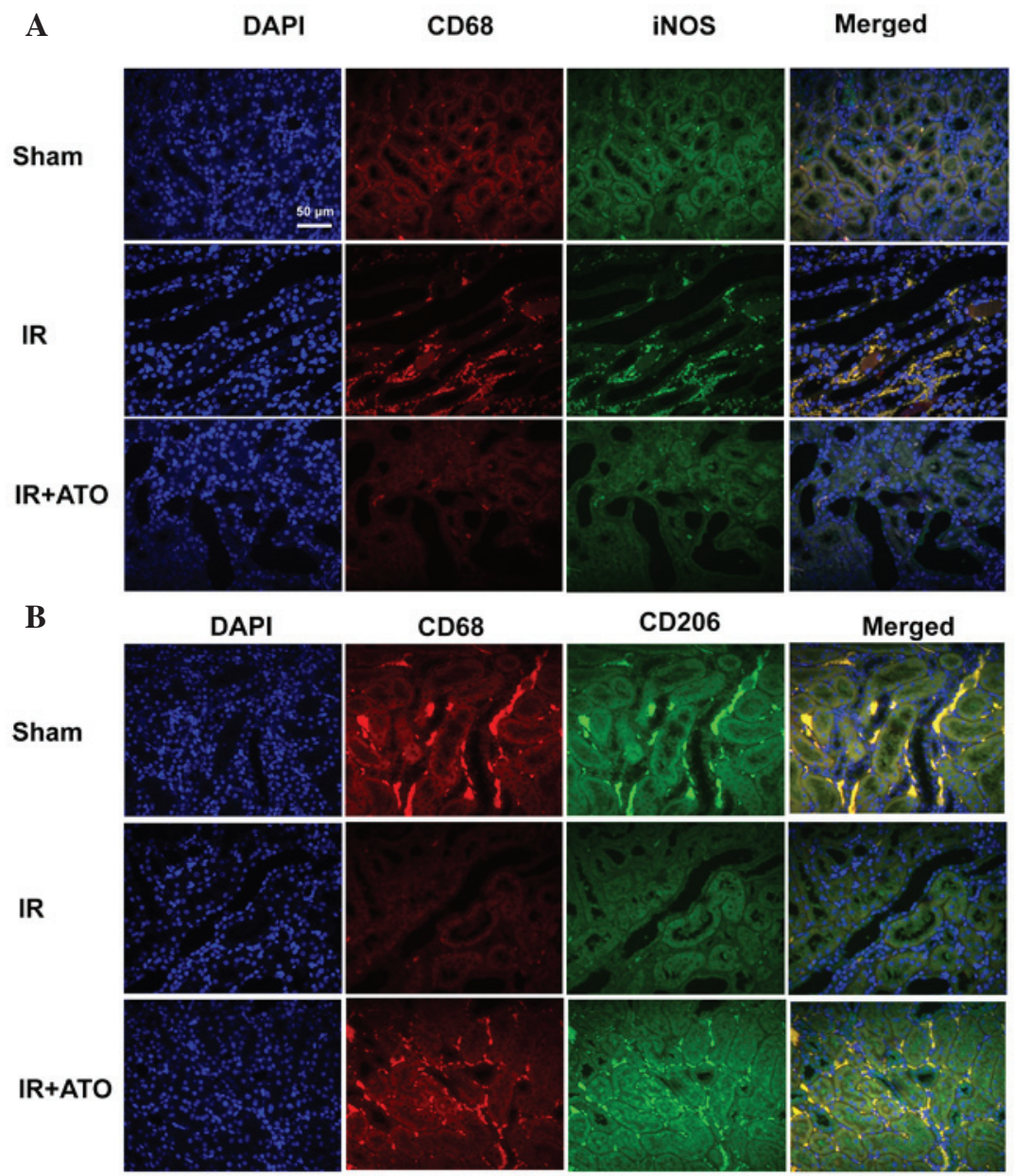

Figure 5. Representative confocal images of indirect double immunofluorescence staining in kidney samples from rats with IR. (A) Double immunofluorescence staining with CD68 and iNOS in the sham, IR and IR + ATO groups. DAPI (blue), CD68 (red), iNOS (green), merged (merged DAPI, CD68 and iNOS). (B) Double immunofluorescence staining with CD68 and CD208 in the sham, IR and IR + ATO groups. DAPI (blue), CD68 (red), CD208 (green), merged (merged DAPI, CD68 and CD208). Scale bar, $50 \mu \mathrm{m}$. IR, ischemia-reperfusion; ATO, atorvastatin; CD, cluster of differentiation; DAPI, 4',6-diamidino-2-phenylindole.

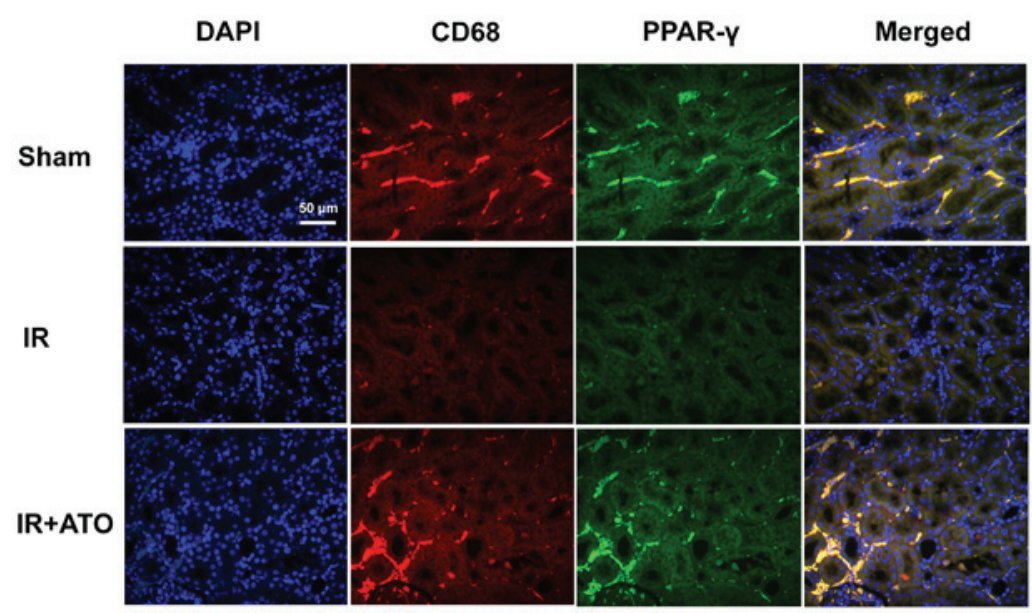

Figure 6. Representative confocal images of CD68 and PPAR- $\gamma$ double immunofluorescence staining in kidney samples from rats with IR. Double immunofluorescence staining with CD68 and PPAR- $\gamma$ in the sham, IR and IR + ATO groups. DAPI (blue), CD68 (red), PPAR- $\gamma$ (green), merged (merged DAPI, CD68 and PPAR- $\gamma$ ). Scale bar, $50 \mu \mathrm{m}$. IR, ischemia-reperfusion; ATO, atorvastatin; CD, cluster of differentiation; DAPI, 4',6-diamidino-2-phenylindole; PPAR- $\gamma$, peroxisome proliferator-activated receptor- $\gamma$.

to the differentiation of monocytes into M2 macrophages, which may functionally contribute to the protection of the kidney from IRI.
The pathophysiology of renal IRI is complex; however, inflammation is a well known associated factor. As a type of statin, ATO has been reported to exert protective effects on 
injuries associated with inflammation, such as hepatic IRI and chronic obstructive pulmonary disease $(14,19)$. The results of the present study revealed that ATO treatment significantly attenuated renal IRI; this finding is consistent with the results of a previous report (15). Furthermore, treatment with ATO significantly decreased IR-induced serum TNF- $\alpha$ and IFN- $\gamma$ levels. It has previously been demonstrated that IFN- $\gamma$, which is associated with macrophage activation and neutrophil recruitment, may amplify the immune response following kidney reperfusion, mediating the early phase of IRI $(2,20)$. Meldrum et al (21) reported that peak serum TNF- $\alpha$ expression occurred after $1 \mathrm{~h}$ of ischemia and $1 \mathrm{~h}$ of reperfusion, and early kidney TNF- $\alpha$ expression is an important mediator of renal IRI (22). These findings suggested that ATO may protect kidneys from IRI by decreasing the levels of TNF- $\alpha$ and IFN- $\gamma$.

Macrophages are an essential component of innate immunity, which have been reported to be critical in the pathogenesis of human renal disease in the glomerulus and renal interstitium (23). A previous study demonstrated that ablation of renal macrophages can improve renal injury in certain models, including IRI, glomerulonephritis and fibrosis (24). It is well known that the macrophage phenotype is regulated by various inflammatory cytokines. M1 macrophage polarization can be induced by $\mathrm{T}$ helper (Th) 1 cytokines, including TNF- $\alpha$ and IFN- $\gamma$, whereas M2 macrophages can be induced by Th2 cytokines, including interleukin (IL)-4 and IL-10. During experimental AKI, there is a spontaneous switch from the M1 to the M2 phenotype (25). Lee et al (26) reported that the proinflammatory M1 phenotype contributes toward IRI early in AKI, whereas the anti-inflammatory M2 phenotype ameliorates injury and stimulates repair. The present study demonstrated that ATO significantly promoted monocyte differentiation toward M2 macrophages and significantly suppressed M1 polarization. These findings suggested that ATO may suppress M1 polarization by inhibiting the inductive effects of TNF- $\alpha$ and IFN- $\gamma$ on monocytes.

As a member of the nuclear hormone receptor family of ligand-dependent transcription factors, PPAR- $\gamma$ has been well characterized as a potent anti-inflammatory factor that modulates the immune inflammatory response (16). Initially described as a master regulator of adipocyte differentiation, PPAR- $\gamma$ has been demonstrated to be highly expressed in macrophages and to functionally contribute to the differentiation of macrophages into M2 macrophages in vitro and in vivo (27-29). Consistent with a previous report that ATO promotes PPAR- $\gamma$ expression in human monocytes (30), the present results indicated that in kidney samples collected following IR, ATO significantly increased PPAR- $\gamma$ expression levels. All these findings indicated that the contributory effects of ATO on M2 differentiation may be mediated by PPAR- $\gamma$.

In conclusion, the present study demonstrated that by promoting M1-M2 differentiation ATO significantly ameliorated AKI after IR. In addition, the findings of the present study suggested that this effect is likely mediated by an increase in PPAR- $\gamma$ expression and a decrease in TNF- $\alpha$ and IFN- $\gamma$ levels. Although the exact underlying mechanism requires further study, the results of the present study suggested that endogenous and exogenous factors, which regulate macrophage phenotype, may affect the occurrence and development of kidney injuries.

\section{Acknowledgements}

The present study was supported by the Guangzhou Medical Key Subject Construction Project (2013-2015; grant no. XM203190).

\section{References}

1. Devarajan P: Emerging biomarkers of acute kidney injury. Contrib Nephrol 156: 203-212, 2007.

2. Kinsey GR, Li L and Okusa MD: Inflammation in acute kidney injury. Nephron Exp Nephrol 109: e102-e107, 2008.

3. Deng J, Kohda Y, Chiao H, Wang Y, Hu X, Hewitt SM, Miyaji T, McLeroy P, Nibhanupudy B, Li S and Star RA: Interleukin-10 inhibits ischemic and cisplatin-induced acute renal injury. Kidney Int 60: 2118-2128, 2001.

4. Kunzendorf U, Haase M, Rolver L and Haase-Fielitz A: Novel aspects of pharmacological therapies for acute renal failure. Drugs 70: 1099-1114, 2010.

5. Geissmann F, Manz MG, Jung S, Sieweke MH, Merad M and Ley K: Development of monocytes, macrophages, and dendritic cells. Science 327: 656-661, 2010.

6. Ginhoux F and Jung S: Monocytes and macrophages: Developmental pathways and tissue homeostasis. Nat Rev Immunol 14: 392-404, 2014.

7. Palmer MB, Vichot AA, Cantley LG and Moeckel GW: Quantification and localization of M2 macrophages in human kidneys with acute tubular injury. Int J Nephrol Renovasc Dis 7: 415-419, 2014.

8. Wang Y and Harris DC: Macrophages in renal disease. J Am Soc Nephrol 22: 21-27, 2011.

9. Hunter MM, Wang A, Parhar KS, Johnston MJ, Van Rooijen N, Beck PL and McKay DM: In vitro-derived alternatively activated macrophages reduce colonic inflammation in mice. Gastroenterology 138: 1395-1405, 2010.

10. Naruszewicz M: Macrophages in the pathogenesis of atherosclerosis. Advances in science and personal observations. Kardiol Pol 32: 27-34, 1989 (In Polish).

11. Olefsky JM and Glass CK: Macrophages, inflammation, and insulin resistance. Annu Rev Physiol 72: 219-246, 2010.

12. Murray PJ and Wynn TA: Protective and pathogenic functions of macrophage subsets. Nat Rev Immunol 11: 723-737, 2011.

13. Sica A and Mantovani A: Macrophage plasticity and polarization: In vivo veritas. J Clin Invest 122: 787-795, 2012.

14. Mroz RM, Lisowski P, Tycinska A, Bierla J, Trzeciak PZ, Minarowski L, Milewski R, Lisowska A, Boros P, Sobkowicz B, et al: Anti-inflammatory effects of atorvastatin treatment in chronic obstructive pulmonary disease. A controlled pilot study. J Physiol Pharmacol 66: 111-128, 2015.

15. Wu K, Lei W, Tian J and Li H: Atorvastatin treatment attenuates renal injury in an experimental model of ischemia-reperfusion in rats. BMC Nephrol 15: 14, 2014.

16. Zhang $\mathrm{O}$ and Zhang J: Atorvastatin promotes human monocyte differentiation toward alternative M2 macrophages through p38 mitogen-activated protein kinase-dependent peroxisome proliferator-activated receptor $\gamma$ activation. Int Immunopharmacol 26: 58-64, 2015.

17. Paller MS, Hoidal JR and Ferris TF: Oxygen free radicals in ischemic acute renal failure in the rat. J Clin Invest 74: 1156-1164, 1984.

18. Xue W, Lei J, Li X and Zhang R: Trigonella foenum graecum seed extract protects kidney function and morphology in diabetic rats via its antioxidant activity. Nutr Res 31: 555-562, 2011.

19. Ghobadi H,Lari SM,Pourfarzi F,Mahmoudpour A and Ghanei M: The effects of atorvastatin on mustard-gas-exposed patients with chronic obstructive pulmonary disease: A randomized controlled trial. J Res Med Sci 19: 99-105, 2014.

20. Dong X, Swaminathan S, Bachman LA, Croatt AJ, Nath KA and Griffin MD: Resident dendritic cells are the predominant TNF-secreting cell in early renal ischemia-reperfusion injury. Kidney Int 71: 619-628, 2007. 
21. Meldrum KK, Meldrum DR, Meng X, Ao L and Harken AH: TNF-alpha-dependent bilateral renal injury is induced by unilateral renal ischemia-reperfusion. Am J Physiol Heart Circ Physiol 282: H540-H546, 2002.

22. Donnahoo KK, Meng X, Ayala A, Cain MP, Harken AH and Meldrum DR: Early kidney TNF-alpha expression mediates neutrophil infiltration and injury after renal ischemia-reperfusion. Am J Physiol 277: R922-R929, 1999.

23. Kluth DC, Erwig LP and Rees AJ: Multiple facets of macrophages in renal injury. Kidney Int 66: 542-557, 2004.

24. Ferenbach DA, Ramdas V, Spencer N, Marson L, Anegon I, Hughes J and Kluth DC: Macrophages expressing heme oxygenase-1 improve renal function in ischemia/reperfusion injury. Mol Ther 18: 1706-1713, 2010.

25. Westenfelder C: Programmed anti-inflammatory macrophages protect against AKI and promote repair through trophic actions. Kidney Int 81: 939-941, 2012.
26. Lee S, Huen S, Nishio H, Nishio S, Lee HK, Choi BS, Ruhrberg C and Cantley LG: Distinct macrophage phenotypes contribute to kidney injury and repair. J Am Soc Nephrol 22: 317-326, 2011.

27. Tontonoz P and Spiegelman BM: Fat and beyond: The diverse biology of PPARgamma. Annu Rev Biochem 77: 289-312, 2008.

28. Penas F, Mirkin GA, Vera M, Cevey Á, González CD, Gómez MI, Sales ME and Goren NB: Treatment in vitro with PPAR $\alpha$ and PPAR $\alpha$ ligands drives M1-to-M2 polarization of macrophages from T. cruzi-infected mice. Biochim Biophys Acta 1852: 893-904, 2015.

29. Pisanu A, Lecca D, Mulas G, Wardas J, Simbula G, Spiga S and Carta AR: Dynamic changes in pro- and anti-inflammatory cytokines in microglia after PPAR- $\alpha$ agonist neuroprotective treatment in the MPTPp mouse model of progressive Parkinson's disease. Neurobiol Dis 71: 280-291, 2014.

30. Grip O, Janciauskiene S and Lindgren S: Atorvastatin activates PPAR-gamma and the inflammatory response in human monocytes. Inflamm Res 51: 58-62, 2002. 